\title{
Lagrangian interactions within a special class of covariant mixed-symmetry type tensor gauge fields
}

\author{
C. Bizdadea* E. M. Cioroianu ${ }^{\dagger}$ I. Negru市 S. O. Saliu ${ }^{\S}$ \\ Faculty of Physics, University of Craiova \\ 13 A. I. Cuza Str., Craiova RO-1100, Romania
}

November 30, 2018

\begin{abstract}
Consistent non-trivial interactions within a special class of covariant mixed-symmetry type tensor gauge fields of degree three are constructed from the deformation of the solution to the master equation combined with specific cohomological techniques. In spacetime dimensions strictly greater than four, the only consistent interaction terms are those gauge invariant under the original symmetry. Only in four spacetime dimensions the gauge symmetry is found to be deformed.
\end{abstract}

PACS number: 11.10.Ef

\section{Introduction}

The cohomological development of the Becchi-Rouet-Stora-Tyutin symmetry, allowed, among others, the determination to be made of the general solution to the Wess-Zumino consistency condition [1]-[10, of the general

*e-mail address: bizdadea@central.ucv.ro

$\dagger$ e-mail address: manache@central.ucv.ro

$\ddagger$ e-mail address: inegru@central.ucv.ro

§e-mail address: osaliu@central.ucv.ro 
form of the counterterms involved with the renormalization of gauge invariant operators (Kluberg-Stern and Zuber conjecture) [6]-[12, the cohomological approach to global symmetries and conservation laws in classical gauge theories [13]-16], the reformulation of the problem of constructing consistent interactions that can be introduced in gauge-invariant theories as a problem of deformation of the solution to the classical master equation [17]-[21], etc. In particular, the cohomological reformulation of consistent interactions in theories with gauge symmetries has led to important results, such as the impossibility of cross-interactions in multigraviton theories [22]-[24], or the existence of the Seiberg-Witten map in noncommutative field theories whose commutative versions allow rigid (i.e. non-deformable) gauge symmetries [25]-[27]. The fundamental algebraic structure on which the BRST symmetry is based is the graded differential complex endowed with a second-order nilpotent differential, together with the (local) cohomology of this differential. Lately, the usual differential tools have been developed to cover generalized differential $N$-complexes [28]-[29] for irreducible tensor gauge fields of mixed Young symmetry type, endowed with higher-order nilpotent operators. In this context, the generalized Poincaré lemma, that governs the cohomology of $N$-complexes related to tensor fields of mixed symmetry type, has been formulated and proved. This modern differential algebraic setting helped at solving some nice problems, like, for instance, the interpretation of the construction of the Pauli-Fierz theory 30, the dual formulation of linearized gravity 31]-32, or the impossibility of consistent cross-interactions in the dual formulation of linearized gravity 32 .

In this paper we investigate all consistent Lagrangian interactions that can be added to a special class of covariant mixed-symmetry type tensor gauge fields of degree three that transform according to a reducible representation of the Lorentz group. Our main results are:

(i) in spacetime dimensions strictly greater than four, the only consistent interactions are gauge invariant terms, that do not deform the gauge symmetry;

(ii) in four dimensions, there appear non-trivial consistent deformations that modify the gauge transformations and their reducibility, but not the gauge algebra.

Our strategy goes as follows. Initially, we generate the antibracketantifield BRST symmetry of the uncoupled model with covariant mixedsymmetry type tensor fields of degree three in an arbitrary spacetime dimension. Next, we apply the deformation procedure and compute the first-order 
deformation of the solution to the master equation, whose integrand belongs to the zeroth-order local cohomology of the free BRST differential $H^{0}(s \mid d)$, where $s$ and $d$ mean the BRST, respectively, the exterior spacetime differentials. Further, we investigate the higher-order deformations. Finally, we analyze the Lagrangian description of the interacting theory, namely, the Lagrangian action, the deformed gauge transformations, and their tensor structure. In $D>4$ spacetime dimensions, the first-order deformation reduces to its antighost number zero component, while all higher-order deformations can be taken to vanish. Thus, the original gauge symmetry is not deformed in this case. For $D=4$, the first-order deformation is parameterized in terms of two arbitrary constants. Its consistency requires that one of the constants must vanish. Under these circumstances, the deformation of the solution to the master equation that is consistent at all orders in the coupling constant ends at the first order. It is local, manifestly Lorentz covariant, and, most important, non-trivial. It follows that the gauge transformations of the mixed-symmetry type tensor fields are truly deformed, although their gauge algebra remains abelian. The interacting model is also first-order reducible, but the reducibility relations hold on-shell, in contrast to the starting uncoupled theory.

This paper is organized into nine sections. Section 2 introduces the Lagrangian model in an arbitrary spacetime dimension. Section 3 is devoted to the BRST symmetry of the uncoupled theory. In Sect. 4 we briefly review the Lagrangian mechanism for constructing consistent interactions from the point of view of the antibracket-antifield BRST method. Section 5 focuses on the deformation procedure in more that four spacetime dimensions and argues that the only consistent interactions simply add gauge-invariant terms to the starting action. In Sect. 6 we restrict ourselves to the four-dimensional case, and reconsider the uncoupled model in first-order form. In this context, in Sect. 7 we solve the main equations of the antibracket-antifield deformation procedure, and show that there are consistent deformations of the solution to the classical master equation at order one in the coupling constant. We investigate the conditions that must be fulfilled such that these are secondand higher-order consistent. In Sect. 8 we identify the Lagrangian version of the interacting model in four dimensions, and analyze the tensor structure of the new gauge theory. Section 9 ends the paper with some conclusions. 


\section{Introducing the free model}

We start from the Lagrangian action for a special class of covariant mixedsymmetry type tensor gauge fields of degree three:

$$
S_{0}\left[A_{\alpha \beta}{ }^{(\sigma)}\right]=-\frac{1}{12} \int d^{D} x F_{\alpha \beta \gamma}{ }^{(\sigma)} F_{(\sigma)}^{\alpha \beta \gamma},
$$

where the fields are only antisymmetric in their first two indices $A_{\alpha \beta}{ }^{(\sigma)}=$ $-A_{\beta \alpha}{ }^{(\sigma)}$. The tensor fields $A_{\alpha \beta}{ }^{(\sigma)}$ can be regarded as being described by a Young diagram with three cells and two columns. However, they do not satisfy the identity $A_{[\alpha \beta(\sigma)]}=0$, associated with the Young symmetrizer of the corresponding diagram. Actually, they transform according to a reducible representation of the Lorentz group. This is the reason why we use the notation $A_{\alpha \beta(\sigma)}$ instead of the standard one, $A_{\alpha \beta \mid \sigma}$. Spacetime indices are raised and lowered with the flat Minkowskian metric of "mostly plus" signature in $D$ dimensions $(D \geq 4):-+++\cdots$. We define the field strength components in the usual manner:

$$
F_{\alpha \beta \gamma}{ }^{(\sigma)}=\partial_{\alpha} A_{\beta \gamma}{ }^{(\sigma)}+\partial_{\beta} A_{\gamma \alpha}{ }^{(\sigma)}+\partial_{\gamma} A_{\alpha \beta}{ }^{(\sigma)} \equiv \partial_{[\alpha} A_{\beta \gamma]}{ }^{(\sigma)},
$$

where we choose the convention that $[\alpha \beta \cdots]$ signifies plain antisymmetry with respect to the indices between brackets, without additional numerical factors. The tensor fields $A_{\alpha \beta}{ }^{(\sigma)}$ can also be viewed in some sense as a collection of two-forms, where the collection index is spacetime-like. This motivates the form (2) of their field strengths, as well as the expression (11) of the Lagrangian action. It is widely known that gauge theories involving antisymmetric tensor fields are important due to their connection with string theory and supergravity models 33-38. In particular, interacting two-forms (described by the Freedman-Townsend model) play a special role due on the one hand to its link with Witten's string theory 39] and, on the other hand, to its equivalence to the nonlinear sigma model [40]. The interacting theory to be derived by us in Sect. 8 resembles in a way the Freedman-Townsend model, but clearly exhibits new features. The gauge invariances of action (11) are given by

$$
\delta_{\epsilon} A_{\alpha \beta}{ }^{(\sigma)}=\partial_{[\alpha} \epsilon_{\beta]}{ }^{(\sigma)} \equiv \stackrel{(0)}{R_{\alpha \beta}} \stackrel{(\sigma) \gamma}{(\sigma)} \epsilon_{\gamma}^{(\rho)},
$$

with $\epsilon_{\gamma}^{(\rho)}$ bosonic gauge parameters, that are tensors of degree two with no symmetry, such that the gauge generators in condensed De Witt notation 
are expressed through

$$
\stackrel{(0)}{R}_{\alpha \beta}^{(\sigma) \gamma} \quad(\rho)=\delta_{\rho}^{\sigma} \partial_{[\alpha} \delta_{\beta]}^{\gamma} .
$$

The above gauge transformations are abelian and turn out to be off-shell first-stage reducible, the accompanying reducibility relations and functions respectively reading

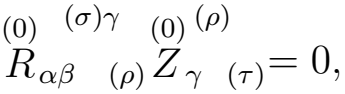

$$
\begin{aligned}
& { }_{(0)}^{(\rho)} \\
& Z_{\gamma(\tau)}=\delta_{\tau}^{\rho} \partial_{\gamma} .
\end{aligned}
$$

In consequence, we deal with a usual free linear gauge theory of Cauchy order three (being local, polynomial in the fields and their derivatives, and satisfying the standard regularity conditions [4], whose gauge algebra is not open, and with a finite reducibility order). As the number of physical degrees

of freedom carried by a tensor field $A_{\alpha \beta}^{(\sigma)}$ is equal to $D(D-2)(D-3) / 2$, the subsequent analysis is meaningful only for $D \geq 4$.

\section{BRST symmetry of the free theory}

We are interested in constructing the consistent Lagrangian (self)interactions that can be added to the action (11) without changing the content of the field spectrum or the number of independent gauge symmetries. In view of this, we apply the general rules of the antibracket-antifield deformation procedure based on (co)homological techniques [17, [21].

The first step in the development of the antibracket-antifield deformation approach consists in generating the Lagrangian BRST symmetry of the free model under study. The Lagrangian BRST complex is organized into the field/ghost, respectively, antifield spectrum

$$
\Phi^{\Delta}=\left(A_{\alpha \beta}^{(\sigma)}, \eta_{\alpha}^{(\sigma)}, C^{(\sigma)}\right) ; \Phi_{\Delta}^{*}=\left(A_{(\sigma)}^{* \alpha \beta}, \eta_{(\sigma)}^{* \alpha}, C_{(\sigma)}^{*}\right),
$$

with the Grassmann parities

$$
\begin{gathered}
\varepsilon\left(A_{\alpha \beta}^{(\sigma)}\right)=0=\varepsilon\left(C^{(\sigma)}\right), \varepsilon\left(\eta_{\alpha}^{(\sigma)}\right)=1, \\
\varepsilon\left(\Phi_{\Delta}^{*}\right)=\left(\varepsilon\left(\Phi^{\Delta}\right)+1\right) \bmod 2 .
\end{gathered}
$$


While the ghosts $\eta_{\alpha}^{(\sigma)}$ are due to the gauge symmetry, the ghosts for ghosts $C^{(\sigma)}$ are required by the first-order reducibility relations. The Lagrangian BRST symmetry acts like a differential $s\left(s^{2}=0\right)$, which we assume to behave like a right derivation. Since the gauge algebra is abelian and the reducibility functions are field independent, it follows that $s$ reduces to the sum of the Koszul-Tate differential $\delta$ and the exterior longitudinal derivative $\gamma$ only,

$$
s=\delta+\gamma,
$$

that are respectively graded in terms of the antighost number (agh) and the pure ghost number (pgh). ${ }^{1}$ While the Koszul-Tate differential $\left(\delta^{2}=0\right.$, $\operatorname{agh}(\delta)=-1, \operatorname{pgh}(\delta)=0)$ realizes a resolution of smooth functions defined on the stationary surface of the field equations, the exterior longitudinal derivative $(\operatorname{pgh}(\gamma)=1, \operatorname{agh}(\gamma)=0)$ anticommutes with $\delta$ and turns out to be a true differential in the particular case of the model under study $\left(\gamma^{2}=0\right)$. Its cohomological space at pure ghost number zero computed in the homology of $\delta, H^{0}\left(\gamma \mid H_{*}(\delta)\right)$, is given by the algebra of the Lagrangian physical observables, and is in the meantime isomorphic to the zeroth order cohomological space of $s, H^{0}(s)$, that contains the so-called BRST observables [47- 49]. The degrees (agh) and (pgh) of the BRST generators (71) are given by

$$
\begin{gathered}
\operatorname{agh}\left(\Phi^{\Delta}\right)=0, \operatorname{agh}\left(A_{(\sigma)}^{* \alpha \beta}\right)=1, \operatorname{agh}\left(\eta_{(\sigma)}^{* \alpha}\right)=2, \operatorname{agh}\left(C_{(\sigma)}^{*}\right)=3, \\
\operatorname{pgh}\left(\Phi_{\Delta}^{*}\right)=0, \operatorname{pgh}\left(A_{\alpha \beta}^{(\sigma)}\right)=0, \operatorname{pgh}\left(\eta_{\alpha}^{(\sigma)}\right)=1, \operatorname{pgh}\left(C^{(\sigma)}\right)=2,
\end{gathered}
$$

while the actions of $\delta$ and $\gamma$ read

$$
\begin{gathered}
\delta \Phi^{\Delta}=0, \delta A_{(\sigma)}^{* \alpha \beta}=-\frac{\delta S_{0}^{L}}{\delta A_{\alpha \beta}^{(\sigma)}} \equiv-\frac{1}{2} \partial_{\gamma} F_{(\sigma)}^{\gamma \alpha \beta}, \\
\delta \eta_{(\sigma)}^{* \alpha}=-2 \partial_{\beta} A_{(\sigma)}^{* \beta \alpha}, \delta C_{(\sigma)}^{*}=\partial_{\alpha} \eta_{(\sigma)}^{* \alpha}, \\
\gamma \Phi_{\Delta}^{*}=0, \gamma A_{\alpha \beta}^{(\sigma)}=\partial_{[\alpha} \eta_{\beta]}^{(\sigma)}, \gamma \eta_{\alpha}^{(\sigma)}=\partial_{\alpha} C^{(\sigma)}, \gamma C^{(\sigma)}=0 .
\end{gathered}
$$

The overall degree of the BRST complex is named ghost number (gh) and is defined like $g h=p g h-$ agh, such that $g h(s)=1$. An important property

\footnotetext{
${ }^{1}$ For more general gauge theories, $s$ has a richer structure than in (10), the addition of supplementary operators $\stackrel{(k)}{s}$, with agh $\left(\begin{array}{c}(k) \\ s\end{array}\right)=k>0$ being necessary, in order to ensure the second-order nilpotency of $s$.
} 
of the Lagrangian BRST symmetry is that it is canonically generated in a structure named antibracket and usually denoted by $($,$) [42 - 49] via a$ generator $\stackrel{(0)}{S}$ :

$$
s \cdot=(\cdot, \stackrel{(0)}{S}) .
$$

The fields/ghosts are decreed conjugated in the antibracket with the associated antifields:

$$
\left(\Phi^{\Delta}, \Phi_{\Delta^{\prime}}^{*}\right)=\delta_{\Delta^{\prime}}^{\Delta}
$$

The second-order nilpotency of $s$ is equivalent to the fact that $\stackrel{(0)}{S}$ is solution to the classical master equation:

$$
(\stackrel{(0)}{S}, \stackrel{(0)}{S})=0, \varepsilon(\stackrel{(0)}{S})=0, \operatorname{gh}\left(\begin{array}{c}
(0) \\
S
\end{array}\right)=0,
$$

with some boundary conditions. In the case of the model investigated here, with the help of the relations (10) and (13) 16), one finds that the complete solution to the master equation can be written in the form

$$
\stackrel{(0)}{S}=S_{0}\left[A_{\alpha \beta}{ }^{(\sigma)}\right]+\int d^{D} x\left(A_{(\sigma)}^{* \alpha \beta} \partial_{[\alpha} \eta_{\beta]}^{(\sigma)}+\eta_{(\sigma)}^{* \alpha} \partial_{\alpha} C^{(\sigma)}\right),
$$

and we observe that it contains pieces of antighost number equal to zero, one, and two, respectively.

\section{Brief review of antibracket-antifield defor- mation procedure}

In order to develop the general approach to the problem of consistent interactions in gauge theories from the perspective of the antibracket-antifield deformation procedure, we briefly recall some basic results [17], 21]. Assume that

$$
\bar{S}_{0}\left[A_{\alpha \beta}{ }^{(\sigma)}\right]=S_{0}\left[A_{\alpha \beta}{ }^{(\sigma)}\right]+g \int d^{D} x a_{0}+O\left(g^{2}\right)
$$

defines a consistent deformation of the action (11), with deformed gauge symmetry

$$
\bar{\delta}_{\epsilon} A_{\alpha \beta}{ }^{(\sigma)}=\bar{R}_{\alpha \beta}{ }^{(\sigma) \gamma}{ }_{(\rho)} \epsilon_{\gamma}{ }^{(\rho)},
$$


where

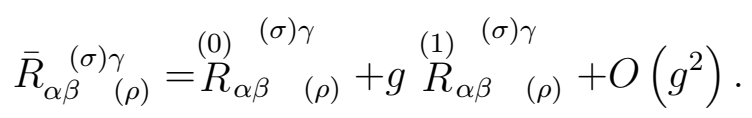

Consistency means that $\bar{S}_{0}$ is fully invariant under the gauge symmetry $\bar{\delta}_{\epsilon}$ (at any order in the coupling constant $g$ ), $\bar{\delta}_{\epsilon} \bar{S}_{0}=0$. Moreover, we add the demand that the number of independent deformed gauge symmetries should remain unchanged with respect to the free theory, which implies the existence of some first-order reducibility functions for the interacting theory,

$$
\bar{Z}_{\gamma(\tau)}^{(\rho)}=\stackrel{(0)}{Z}_{\gamma}^{(\rho)}(\tau)+g \stackrel{(1)}{Z}_{\gamma(\tau)}^{(\rho)}+O\left(g^{2}\right)
$$

such that the new reducibility relations may now hold on-shell, i.e., on the stationary surface of field equations for $\bar{S}_{0}$ :

$$
\bar{R}_{\alpha \beta}^{(\sigma) \gamma(\rho)} \bar{Z}_{\gamma(\tau)}^{(\rho)} \approx 0
$$

It is possible to reformulate more economically this problem in terms of the solution to the master equation. The key observation on which this approach relies is that a consistent deformation of the free action (11) and of its gauge symmetries (3) defines a deformation of the solution (19) of the master equation that preserves both the master equation and the field/ghost-antifield spectrum. Indeed, if the interactions can be consistently constructed, then the solution (19) of the master equation for the free theory can be deformed into the solution $\bar{S}$ of the master equation for the interacting theory, thus:

$$
\begin{gathered}
\stackrel{(0)}{S} \rightarrow \bar{S}=\stackrel{(0)}{S}+g \stackrel{(1)}{S}+g^{2} \stackrel{(2)}{S}+\cdots, \\
(\stackrel{(0)}{S}, \stackrel{(0)}{S})=0 \rightarrow(\bar{S}, \bar{S})=0 .
\end{gathered}
$$

The master equation $(\bar{S}, \bar{S})=0$ guarantees that the consistency requirements on $\bar{S}_{0}, \bar{R}_{\alpha \beta}{ }_{\alpha(\rho) \gamma}(\rho)$ and $\bar{Z}_{\gamma}^{(\rho)}(\tau)$ are fulfilled. The main advantage in reformulating the problem of consistent interactions as the problem of deforming the master equation is that we can make use of the cohomological techniques of the deformation theory. The master equation for $\bar{S}$ splits according to the deformation parameter $g$ as

$$
g^{0}:(\stackrel{(0)}{S}, \stackrel{(0)}{S})=0
$$




$$
\begin{gathered}
g^{1}: 2(\stackrel{(1)}{S}, \stackrel{(0)}{S})=0 \\
g^{2}: 2(\stackrel{(2)}{S}, \stackrel{(0)}{S})+(\stackrel{(1)}{S}, \stackrel{(1)}{S})=0,
\end{gathered}
$$

Equation (27) is checked by assumption, while (28) stipulates that the firstorder deformation is a cocycle of the free BRST differential (16),

$$
s \stackrel{(1)}{S}=0 .
$$

However, only cohomologically non-trivial solutions to (28) should be taken into account, since trivial (BRST-exact) ones can be eliminated by a (in general non-linear) field redefinition [21. In this way, we conclude that the non-trivial deformations $\stackrel{(1)}{S}$ are determined by the zeroth-order cohomological space $H^{0}(s)$ of the undeformed theory, which is generically non-empty due to its isomorphism to the space of physical observables of the free theory. The next equation, (29), implies that $(\stackrel{(1)}{S}, \stackrel{(1)}{S})$ must be trivial (BRST-exact) in the cohomology of $s$ at ghost number one, $H^{1}(s)$. There are no obstructions in finding solutions to the remaining equations (29), etc. (for instance, see [21])) as long as no restrictions on the interactions, such as spacetime locality or manifest Lorentz covariance, are imposed. In general, the resulting interactions may be nonlocal, and there might even appear obstructions if one insists on their locality. However, as it will be seen below, in the case of the model under consideration we obtain only local and manifestly covariant interactions.

\section{Deformations in $D>4$}

There are three different types of deformations, namely:

(i) that modify only the Lagrangian action, but not the gauge symmetry;

(ii) that change the gauge symmetries, but not their algebra; and

(iii) that deform also the gauge algebra. In spacetime dimensions strictly greater than four $(D>4)$, the only consistent non-trivial deformations for the model under study are shown to be only of type (i), and hence they 
merely add to the free action (11) interaction terms that are invariant under the original gauge transformations (3). This can be seen by developing the first-order deformation of the solution to the master equation according to the antighost number

$$
\stackrel{(1)}{S}=\sum_{k=0}^{n} \int d^{D} x a_{k}, \operatorname{gh}\left(a_{k}\right)=0, \varepsilon\left(a_{k}\right)=0, \operatorname{agh}\left(a_{k}\right)=k .
$$

The equation for $\stackrel{(1)}{S}$, namely (30) takes the local form

$$
s\left(\sum_{k=0}^{n} a_{k}\right)=\partial_{\mu} h^{\mu}
$$

The number of terms from the expansion of $\stackrel{(1)}{S}$ is finite and it can be shown that one can take the piece of highest antighost number to be $\gamma$-closed, $\gamma a_{n}=0$, and so $a_{n} \in H(\gamma)$. This can be done by using the arguments from [7, 23], 41], 50]. From the definitions (13) 15), it is simple to see that $H(\gamma)$ is generated by the field strength components $F_{\alpha \beta \gamma}{ }^{(\sigma)}$ and their derivatives, by the antifields $\Phi_{\Delta}^{*}$ together with their derivatives, as well as by the undifferentiated ghosts for ghosts $C^{(\sigma)}$. Since gh $\left(C^{(\sigma)}\right)=2$, it follows that $n=2 m$ and

$$
a_{n} \equiv a_{2 m}=\mu^{\rho_{1} \cdots \rho_{m}}\left(\left[\Phi_{\Delta}^{*}\right],\left[F_{\alpha \beta \gamma}{ }^{(\sigma)}\right]\right) C_{\left(\rho_{1}\right)} \cdots C_{\left(\rho_{m}\right)},
$$

where the notation $f([q])$ signifies that $f$ depends on $q$ and its derivatives up to a finite order. The spacetime derivatives of the ghosts for ghosts are exact in $H(\gamma)$ according to the third relation in (15), and hence trivial, such that they are discarded from $a_{2 m}$. By projecting (32) on antighost number $(2 m-1)$, we infer the equation $\delta a_{2 m}+\gamma a_{2 m-1}=\partial_{\mu} h^{\prime \mu}$. Replacing the expression (33) in the last equation, we find the result that a necessary condition for the existence of $a_{2 m-1}$ is that the functions $\mu^{\rho_{1} \cdots \rho_{m}}$ belong to the local homology of the Koszul-Tate differential at antighost number $2 m$, $\mu^{\rho_{1} \cdots \rho_{m}} \in H_{2 m}(\delta \mid d)$,

$$
\delta \mu^{\rho_{1} \cdots \rho_{m}}=\partial_{\mu} h^{\prime \mu \rho_{1} \cdots \rho_{m}}, \operatorname{agh}\left(\mu^{\rho_{1} \cdots \rho_{m}}\right)=2 m, \operatorname{pgh}\left(\mu^{\rho_{1} \cdots \rho_{m}}\right)=0 .
$$

But as the model under study is linear and of Cauchy order equal to three, we have the local homology of $\delta$ vanishing for antighost numbers greater 
that three, $H_{k}(\delta \mid d)=0, k>3$. On the other hand, the last representative, $a_{n}$, is constructed from some functions that pertain to an even-order space $H_{2 m}(\delta \mid d)$, such that the first admitted value $2 m \leq 3$ is $m=1(n=2)$. Then we can assume that $\stackrel{(1)}{S}=\sum_{k=0}^{2} \int d^{D} x a_{k}$, where $a_{2}=\mu^{\rho} C_{(\rho)}$, with $\mu^{\rho} \in$ $H_{2}(\delta \mid d)$.

At this stage, we remark that the general representative of $H_{2}(\delta \mid d)$ is of the form $\alpha=\lambda_{\alpha}^{(\sigma)} \eta_{(\sigma)}^{* \alpha}$, for some constants $\lambda_{\alpha}^{(\sigma)}$. Consequently, it follows that $\mu^{\rho}=\lambda^{\sigma} \eta_{(\sigma)}^{* \rho}+\tilde{\lambda}_{\alpha} \eta^{* \alpha(\rho)}$, where $\lambda^{\sigma}$ and $\tilde{\lambda}_{\alpha}$ are also constant. On the other hand, the only covariant choice of these constants is $\lambda^{\sigma}=k_{1} \partial^{\sigma}$ and $\tilde{\lambda}_{\alpha}=k_{2} \partial_{\alpha}$, which further yields

$$
\mu^{\rho}=k_{1} \partial^{\sigma} \eta_{(\sigma)}^{* \rho}+k_{2} \partial_{\alpha} \eta^{* \alpha(\rho)},
$$

with $k_{1,2}$ numerical constants. The second term on the right hand-side of (35) is $\delta$-exact (see the latter relations in (14)). As one can always add a $\delta$-exact term to the solution of the equation $\delta \mu^{\rho}=\partial_{\alpha} h^{\prime \alpha \rho}$, we have the result that the second term from the right hand-side of (35) can be removed. Then we find that

$$
a_{2}=\partial^{\sigma}\left(k_{1} \eta_{(\sigma)}^{* \rho} C_{(\rho)}\right)-\gamma\left(k_{1} \eta_{(\sigma)}^{* \rho} \eta_{(\rho)}^{\sigma}\right) .
$$

Since we are free to add a $\gamma$-exact term and a $\gamma$-invariant divergence to the last representative, we get the result that $a_{2}$ can be chosen to vanish, $a_{2}=0$. Further, $a_{1}$ is not eligible as the last representative in $\stackrel{(1)}{S}$ because it does not display an even pure ghost number. As a consequence, $\stackrel{(1)}{S}$ simply reduces to the component that is ghost and antifield independent, $\stackrel{(1)}{S}=\int d^{D} x a_{0}$, where $a_{0}$ is a $\gamma$-cocycle modulo $d, \gamma a_{0}=\partial_{\mu} h^{\prime \prime \mu}$. The non-trivial solutions to these equations are of two kinds. The first one corresponds to $h^{\prime \prime \mu}=0$ and is given by local functions that are invariant under the original gauge transformations, which are polynomials in the field strength components $F_{\alpha \beta \gamma}{ }^{(\sigma)}$ and their spacetime derivatives, while the second kind is associated with $h^{\prime \prime \mu} \neq 0$ and is spanned by generalized Chern-Simons terms.

In conclusion, the only non-trivial first-order deformation of the solution to the master equation for a special class of mixed-symmetry type tensor gauge fields of degree three in spacetime dimensions strictly greater than four is, according to the classification from the beginning of this section, of type (i). Beside the Lagrangian action, neither the gauge algebra, nor the gauge transformations, nor the reducibility relations are modified. The 
deformed solution $\bar{S}=\stackrel{(0)}{S}+g \int d^{D} x a_{0}$ is already consistent to all orders in the deformation parameter, so we can take $\stackrel{(k)}{S}=0, k>1$.

\section{Four-dimensional model in first-order form}

Since there are no deformations of the free solution (19) to the master equation in more that four spacetime dimensions that truly deform the gauge transformations and eventually their algebra, in the sequel we focus on the four- dimensional case. It is convenient to rewrite the Lagrangian action (11) for $D=4$ in first-order form. For subsequent purpose, we replace the tensor fields $A_{\alpha \beta}{ }^{(\sigma)}$ by their duals with respect to the antisymmetry indices, $\frac{1}{2} \varepsilon^{\alpha \beta \gamma \delta} A_{\gamma \delta(\sigma)}$, which we will still denote by $A_{(\sigma)}^{\alpha \beta}$, so the Lagrangian action in first-order form becomes

$$
S_{0}^{\prime}\left[A_{(\sigma)}^{\alpha \beta}, B_{\alpha}^{(\sigma)}\right]=\frac{1}{2} \int d^{4} x\left(-B_{\alpha}^{(\sigma)} B_{(\sigma)}^{\alpha}+A_{(\sigma)}^{\alpha \beta} H_{\alpha \beta}{ }^{(\sigma)}\right),
$$

by means of adding an auxiliary tensor field of degree two $B_{\alpha}^{(\sigma)}$ with no symmetry, endowed with the field strength

$$
H_{\alpha \beta}^{(\sigma)}=\partial_{\alpha} B_{\beta}^{(\sigma)}-\partial_{\beta} B_{\alpha}^{(\sigma)} \equiv \partial_{[\alpha} B_{\beta]}^{(\sigma)} .
$$

It is known that auxiliary fields do not change the dynamics, and, essentially, they do not modify the local cohomological group $H^{0}(s \mid d)$ either [41]. The gauge invariances of action (37) are given by

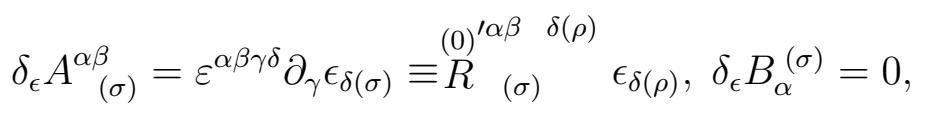

with

$$
\stackrel{(0)}{R}_{(\sigma)}^{\prime \alpha \beta} \quad \delta(\rho)=\delta_{\sigma}^{\rho} \varepsilon^{\alpha \beta \gamma \delta} \partial_{\gamma},
$$

and $\varepsilon^{\alpha \beta \gamma \delta}$ is the completely antisymmetric symbol in four spacetime dimensions, $\varepsilon^{0123}=+1$. They are off-shell first-stage reducible, where the reducibility relations and functions read

$$
\stackrel{(0)}{R}_{(\sigma)}^{\alpha \beta} \stackrel{\delta(\rho)}{(0)} \stackrel{(}{Z}_{\delta(\rho)}^{\prime}=0
$$




$$
\stackrel{(0)}{Z}_{\delta(\rho)}^{(\tau)}=\delta_{\rho}^{\tau} \partial_{\delta} .
$$

The generators of the BRST complex and their degrees are listed below:

$$
\begin{gathered}
\Phi^{\Delta}=\left(A_{(\sigma)}^{\alpha \beta}, B_{\alpha}^{(\sigma)}, \eta_{\alpha(\sigma)}, C_{(\sigma)}\right), \\
\Phi_{\Delta}^{*}=\left(A_{\alpha \beta}^{*(\sigma)}, B_{(\sigma)}^{* \alpha}, \eta^{* \alpha(\sigma)}, C^{*(\sigma)}\right), \\
\varepsilon\left(A_{(\sigma)}^{\alpha \beta}\right)=\varepsilon\left(B_{\alpha}^{(\sigma)}\right)=\varepsilon\left(C_{(\sigma)}\right)=0, \varepsilon\left(\eta_{\alpha(\sigma)}\right)=1, \\
\operatorname{agh}\left(\Phi^{\Delta}\right)=0, \operatorname{agh}\left(A_{\alpha \beta}^{*(\sigma)}\right)=1=\operatorname{agh}\left(B_{(\sigma)}^{* \alpha}\right), \\
\operatorname{agh}\left(\eta^{* \alpha(\sigma)}\right)=2, \operatorname{agh}\left(C^{*(\sigma)}\right)=3, \\
\operatorname{pgh}\left(\Phi_{\Delta}^{*}\right)=0, \operatorname{pgh}\left(A_{(\sigma)}^{\alpha \beta}\right)=0=\operatorname{pgh}\left(B_{\alpha}^{(\sigma)}\right), \\
\operatorname{pgh}\left(\eta_{\alpha(\sigma)}\right)=1, \operatorname{pgh}\left(C_{(\sigma)}\right)=2 .
\end{gathered}
$$

In terms of the new variables, the BRST differential $s=\delta+\gamma$ of the free theory has the form

$$
\begin{gathered}
\delta \Phi^{\Delta}=0, \delta A_{\alpha \beta}^{*(\sigma)}=-\frac{1}{2} H_{\alpha \beta}^{(\sigma)}, \delta B_{(\sigma)}^{* \alpha}=B_{(\sigma)}^{\alpha}+\partial_{\beta} A_{(\sigma)}^{\beta \alpha}, \\
\delta \eta^{* \alpha(\sigma)}=\varepsilon^{\alpha \beta \gamma \delta} \partial_{\beta} A_{\gamma \delta}^{*(\sigma)}, \delta C^{*(\sigma)}=\partial_{\alpha} \eta^{* \alpha(\sigma)}, \\
\gamma \Phi_{\Delta}^{*}=0, \gamma A_{(\sigma)}^{\alpha \beta}=\varepsilon^{\alpha \beta \gamma \delta} \partial_{\gamma} \eta_{\delta(\sigma)}, \gamma\left(B_{\alpha}^{(\sigma)}\right)=0, \\
\gamma \eta_{\alpha(\sigma)}=\partial_{\alpha} C_{(\sigma)}, \gamma C_{(\sigma)}=0,
\end{gathered}
$$

whereas the solution to the classical master equation for the free model with auxiliary fields is

$$
\stackrel{(0)}{S}=S_{0}^{\prime}\left[A_{(\sigma)}^{\alpha \beta}, B_{\alpha}^{(\sigma)}\right]+\int d^{4} x\left(\varepsilon^{\alpha \beta \gamma \delta} A_{\alpha \beta}^{*(\sigma)} \partial_{\gamma} \eta_{\delta(\sigma)}+\eta^{* \alpha(\sigma)} \partial_{\alpha} C_{(\sigma)}\right),
$$

being understood that $s \cdot=(\cdot, \stackrel{(0)}{S})$. 


\section{Lagrangian BRST deformation in four di- mensions}

We have seen that (28) requires that the first-order deformation of the solution to the master equation is an $s$-cocycle modulo $d$ at ghost number zero

$$
\stackrel{(1)}{S}=\int d^{4} x a, s \stackrel{(1)}{S}=0 \Leftrightarrow s a=\partial_{\mu} u^{\mu}
$$

In four dimensions, on the one hand the local homology of $\delta$ vanishes again for $k>3$ [20],

$$
H_{k}(\delta \mid d)=0, k>3,
$$

and, on the other hand, the cohomology of $\gamma$ is generated by $\left(\Phi_{\Delta}^{*}, B_{\alpha}^{(\sigma)}\right)$ and their spacetime derivatives up to some finite orders, together with the undifferentiated ghosts for ghosts $C_{(\sigma)}$. (We did not include the field strength components (2) or their derivatives in $H(\gamma)$ because the auxiliary fields and their derivatives take over this role via the equations of motion for $B_{\alpha}^{(\sigma)}$.) As discussed in the previous section, the cocycle $a$ can be assumed to decompose in a finite number of terms via the antighost number

$$
a=\sum_{k=0}^{n} a_{k}, \operatorname{gh}\left(a_{k}\right)=0, \operatorname{agh}\left(a_{k}\right)=k,
$$

where the component of highest antighost number belongs to $H(\gamma)$ :

$$
\gamma a_{n}=0
$$

Following a reasoning similar to that of Sect. 5 , we infer that $n=2 m$ and

$$
a_{2 m}=\mu^{\rho_{1} \cdots \rho_{m}}\left(\left[\Phi_{\Delta}^{*}\right],\left[B_{\alpha}^{(\sigma)}\right]\right) C_{\left(\rho_{1}\right)} \cdots C_{\left(\rho_{m}\right)},
$$

with $\operatorname{agh}\left(\mu^{\rho_{1} \cdots \rho_{m}}\right)=2 m, \operatorname{pgh}\left(\mu^{\rho_{1} \cdots \rho_{m}}\right)=0$. In the meantime, the local form of the equation (55) projected on antighost number $(2 m-1)$,

$$
\delta a_{2 m}+\gamma a_{2 m-1}=\partial_{\mu} v^{\mu}
$$

induces the result that a necessary condition for the existence of $a_{2 m-1}$ is that

$$
\mu^{\rho_{1} \cdots \rho_{m}} \in H_{2 m}(\delta \mid d)
$$


Combining (56) with (59), it is legitimate to presume that the expansion (57) stops after the first three terms $(n=2 m=2)$,

$$
a=a_{0}+a_{1}+a_{2}
$$

where $a_{2}$ is of the form $\mu^{\rho} C_{(\rho)}$, with $\mu^{\rho}$ an element of $H_{2}(\delta \mid d)$, whose dependence on the fields and antifields is like in (59). After some computation, we find that $H_{2}(\delta \mid d)$ is two-dimensional, where a possible choice of its two independent and non-trivial elements is

$$
\begin{aligned}
& \mu^{\prime \rho}=-\left(\partial_{\sigma} \eta^{* \alpha(\rho)}\right) B_{\alpha}^{(\sigma)}+\eta^{* \alpha(\sigma)} \partial_{\sigma} B_{\alpha}^{(\rho)}+\varepsilon^{\alpha \beta \gamma \delta} A_{\alpha \beta}^{*{ }^{(\sigma)}} \partial_{\sigma} A_{\gamma \delta}^{*}{ }^{(\rho)}, \\
& \mu^{\prime \prime \rho}=-\left(\partial^{\rho} \eta^{* \alpha(\sigma)}\right) B_{\alpha(\sigma)}+\eta^{* \alpha(\sigma)} \partial^{\rho} B_{\alpha(\sigma)}+\varepsilon^{\alpha \beta \gamma \delta} A_{\alpha \beta}^{*{ }^{(\sigma)}} \partial^{\rho} A_{\gamma \delta(\sigma)}^{*} .
\end{aligned}
$$

Indeed, we have

$$
\begin{aligned}
\delta \mu^{\prime \rho} & =\partial_{\mu}\left(\varepsilon^{\mu \alpha \beta \gamma}\left(B_{\gamma}^{(\sigma)} \partial_{\sigma} A_{\alpha \beta}^{*}{ }^{(\rho)}-A_{\alpha \beta}^{*}{ }^{(\sigma)} \partial_{\sigma} B_{\gamma}^{(\rho)}\right)\right), \\
\delta \mu^{\prime \prime \rho} & =\partial_{\mu}\left(\varepsilon^{\mu \alpha \beta \gamma}\left(B_{\gamma}^{(\sigma)} \partial^{\rho} A_{\alpha \beta(\sigma)}^{*}-A_{\alpha \beta}^{*(\sigma)} \partial^{\rho} B_{\gamma(\sigma)}\right)\right) .
\end{aligned}
$$

Consequently, the last component in the first-order deformation (62) takes the concrete form

$$
\begin{aligned}
& a_{2}=\left(-\left(c_{1} \partial_{\sigma} \eta^{* \alpha(\rho)}+c_{2} \partial^{\rho} \eta_{(\sigma)}^{* \alpha}\right) B_{\alpha}^{(\sigma)}+\right. \\
& \eta^{* \alpha(\sigma)}\left(c_{1} \partial_{\sigma} B_{\alpha}^{(\rho)}+c_{2} \partial^{\rho} B_{\alpha(\sigma)}\right)+ \\
& \left.\varepsilon^{\alpha \beta \gamma \delta} A_{\alpha \beta}^{*(\sigma)}\left(c_{1} \partial_{\sigma} A_{\gamma \delta}^{*(\rho)}+c_{2} \partial^{\rho} A_{\gamma \delta(\sigma)}^{*}\right)\right) C_{(\rho)},
\end{aligned}
$$

with $c_{1}$ and $c_{2}$ two real constants, and otherwise arbitrary. From the action of $\delta$ on $a_{2}$,

$$
\begin{aligned}
& \delta a_{2}=-\gamma\left(\varepsilon ^ { \alpha \beta \gamma \delta } \left(A_{\alpha \beta}^{*(\sigma)}\left(c_{1} \partial_{\sigma} B_{\gamma}^{(\rho)}+c_{2} \partial^{\rho} B_{\gamma(\sigma)}\right)-\right.\right. \\
& \left.\left.\left(c_{1} \partial_{\sigma} A_{\alpha \beta}^{*(\rho)}+c_{2} \partial^{\rho} A_{\alpha \beta(\sigma)}^{*}\right) B_{\gamma}^{(\sigma)}\right) \eta_{\delta(\rho)}\right)+ \\
& \partial_{\mu}\left(\varepsilon ^ { \mu \alpha \beta \gamma } \left(B_{\gamma}^{(\sigma)}\left(c_{1} \partial_{\sigma} A_{\alpha \beta}^{*(\rho)}+c_{2} \partial^{\rho} A_{\alpha \beta(\sigma)}^{*}\right)-\right.\right. \\
& \left.\left.-A_{\alpha \beta}^{*(\sigma)}\left(c_{1} \partial_{\sigma} B_{\gamma}^{(\rho)}+c_{2} \partial^{\rho} B_{\gamma(\sigma)}\right)\right) C_{(\rho)}\right)
\end{aligned}
$$

(60) for $m=1$ yields $a_{1}$ :

$$
\begin{aligned}
& a_{1}=\varepsilon^{\alpha \beta \gamma \delta}\left(A_{\alpha \beta}^{*(\sigma)}\left(c_{1} \partial_{\sigma} B_{\gamma}^{(\rho)}+c_{2} \partial^{\rho} B_{\gamma(\sigma)}\right)-\right. \\
& \left.\left(c_{1} \partial_{\sigma} A_{\alpha \beta}^{*(\rho)}+c_{2} \partial^{\rho} A_{\alpha \beta(\sigma)}^{*}\right) B_{\gamma}^{(\sigma)}\right) \eta_{\delta(\rho)},
\end{aligned}
$$


up to a solution $a_{1}^{\prime}$ of the "homogeneous" equation $\gamma a_{1}^{\prime}=\partial_{\mu} k^{\mu}$ and, certainly, up to trivial irrelevant terms. The solutions of the homogeneous equation do modify the gauge transformations, but not their algebra, since they correspond to a vanishing $a_{2}$. Using again the arguments from [7, [23, 41], [50, it can be shown that we can redefine $a_{1}^{\prime}$ such that $a_{1}^{\prime}$ is a $\gamma$-cocycle, $\gamma a_{1}^{\prime}=0$. As $\operatorname{pgh}\left(a_{1}^{\prime}\right)=1$, it follows that it must be linear in the ghosts $\eta_{\delta(\rho)}$ and their spacetime derivatives. However, on account of the relations (52 53) we observe that neither the ghosts nor their derivatives are non-trivial in $H(\gamma)$, and hence we can take $a_{1}^{\prime}=0$.

At this point we are able to determine the term $a_{0}$, which is precisely the deformed Lagrangian at order one in $g$. The equation for $a_{0}$ follows from (55) (with $a$ in the form (62) ) projected on antighost number zero:

$$
\delta a_{1}+\gamma a_{0}=\partial_{\mu} w^{\mu}
$$

By means of (69), we find that

$$
\begin{aligned}
& \delta a_{1}=\gamma\left(\left(c_{1} \partial_{\sigma} B_{\alpha}^{(\rho)}+c_{2} \partial^{\rho} B_{\alpha(\sigma)}\right) B_{\beta}^{(\sigma)} A_{(\rho)}^{\alpha \beta}\right)- \\
& \partial_{\mu}\left(\varepsilon^{\mu \alpha \beta \gamma}\left(c_{1} \partial_{\sigma} B_{\alpha}^{(\rho)}+c_{2} \partial^{\rho} B_{\alpha(\sigma)}\right) B_{\beta}^{(\sigma)} \eta_{\gamma(\rho)}\right),
\end{aligned}
$$

which further leads to the expression

$$
a_{0}=-\left(c_{1} \partial_{\sigma} B_{\alpha}^{(\rho)}+c_{2} \partial^{\rho} B_{\alpha(\sigma)}\right) B_{\beta}^{(\sigma)} A_{(\rho)}^{\alpha \beta},
$$

also up to a solution $a_{0}^{\prime}$ of the "homogeneous" equation $\gamma a_{0}^{\prime}=\partial_{\mu} l^{\mu}$ corresponding to vanishing $a_{1}$. These solutions only modify the free action (37) by adding to it terms that are invariant under the gauge transformations (39) of the free model, $\gamma\left(\int d^{4} x a_{0}^{\prime}\right)=0$. The most general form of such gauge-invariant terms is expressed by arbitrary polynomials in the auxiliary fields $B_{\alpha}^{(\sigma)}$ and their derivatives. However, these interactions are less interesting as they merely change the field equations, but do not modify the gauge symmetry.

Now, the first-order deformation of the solution to the master equation

$$
\stackrel{(1)}{S}=\int d^{4} x a=\int d^{4} x\left(a_{0}+a_{1}+a_{2}\right)
$$

where $a_{0,1,2}$ are indicated in (67), (69) and (72), is by construction an $s$ cocycle of ghost number zero, such that $\stackrel{(0)}{S}+g \stackrel{(1)}{S}$ is a solution to the master 
equation up to order $g$. It is essential to remark that the interactions are not trivial, and that they truly deform the gauge symmetry and its reducibility, since the antifields cannot be eliminated from $a$ even by redefinitions.

Next, let us investigate the consistency of the first-order deformation at order $g^{2}$. In this light, we invoke (29). If we employ the notations $\stackrel{(2)}{S}=\int d^{4} x b$ and $(1 / 2)(\stackrel{(1)}{S}, \stackrel{(1)}{S})=\int d^{4} x \Delta$, then (29) takes the local form

$$
\Delta=-s b+\partial_{\mu} f^{\mu}
$$

By direct computation, we get

$$
\begin{aligned}
& \Delta=\left(c_{1}\right)^{2} \varepsilon^{\alpha \beta \gamma \delta} \partial_{\mu}\left(B_{\alpha}^{(\mu)}\left(B_{\beta}^{(\sigma)}\left(\partial_{\sigma} B_{\gamma}^{(\rho)}\right) \eta_{\delta(\rho)}-A_{\beta \gamma}^{*(\sigma)}\left(\partial_{\sigma} B_{\delta}^{(\rho)}\right) C_{(\rho)}\right)+\right. \\
& \left.\left(B_{\alpha}^{(\mu)}\left(\partial_{\rho} A_{\beta \gamma}^{*(\sigma)}\right) B_{\delta}^{(\rho)}-A_{\alpha \beta}^{*(\mu)}\left(\partial_{\rho} B_{\gamma}^{(\sigma)}\right) B_{\delta}^{(\rho)}\right) C_{(\sigma)}\right)+ \\
& c_{2} \varepsilon^{\alpha \beta \gamma \delta}\left(\partial_{\sigma} B_{\gamma}^{(\rho)}\right)\left(c_{1} A_{\alpha \beta}^{*(\sigma)}\left(2\left(\partial^{\tau} B_{\delta(\rho)}\right) C_{(\tau)}+B_{\delta(\rho)} \partial^{\tau} C_{(\tau)}\right)-\right. \\
& \left.c_{2}\left(2\left(\partial^{\tau} A_{\alpha \beta}^{*(\sigma)}\right) C_{(\tau)}+A_{\alpha \beta}^{*}{ }^{(\sigma)} \partial^{\tau} C_{(\tau)}\right) B_{\delta(\rho)}\right)+ \\
& c_{1} c_{2} \varepsilon^{\alpha \beta \gamma \delta}\left(-B_{\delta(\rho)}\left(\partial^{\tau} B_{\gamma}^{(\rho)}\right) \partial_{\sigma}\left(A_{\alpha \beta}^{*(\sigma)} C_{(\tau)}\right)+\right. \\
& \left(\left(\partial^{\rho} A_{\alpha \beta}^{*}(\tau)\right.\right. \\
& \left.\left(\partial_{\rho} B_{\delta(\sigma)}\right)-\left(\partial^{\rho} A_{\alpha \beta(\sigma)}^{*}\right)\left(\partial_{\rho} B_{\delta}^{(\tau)}\right)\right) B_{\gamma}^{(\sigma)} C_{(\tau)}+ \\
& \left(A_{\alpha \beta(\sigma)}^{*} \partial^{\tau} B_{\gamma}^{(\sigma)}-\left(\partial^{\tau} A_{\alpha \beta(\sigma)}^{*}\right) B_{\gamma}^{(\sigma)}\right) \partial^{\rho}\left(B_{\delta(\rho)} C_{(\tau)}\right)+ \\
& +\left(\partial^{\rho} A_{\alpha \beta(\sigma)}^{*}\right)\left(2\left(\partial^{\tau} B_{\gamma}^{(\sigma)}\right) C_{(\tau)}+B_{\gamma}^{(\sigma)} \partial^{\tau} C_{(\tau)}\right) B_{\delta(\rho)}- \\
& \left(2\left(\partial^{\tau} A_{\alpha \beta(\sigma)}^{*}\right) C_{(\tau)}+A_{\alpha \beta(\sigma)}^{*} \partial^{\tau} C_{(\tau)}\right)\left(\partial^{\rho} B_{\gamma}^{(\sigma)}\right) B_{\delta(\rho)}+ \\
& A_{\alpha \beta(\sigma)}^{*}\left(\partial^{\rho} B_{\gamma}^{(\sigma)}\right)\left(\partial_{\rho} B_{\delta}^{(\tau)}\right) C_{(\tau)}-B_{\gamma(\rho)}\left(\partial^{\rho} B_{\beta(\sigma)}\right) \partial^{\tau}\left(B_{\alpha}^{(\sigma)} \eta_{\delta(\tau)}\right)+ \\
& \left.B_{\alpha}^{(\sigma)}\left(\left(\partial_{\sigma} B_{\beta}^{(\rho)}+\partial^{\rho} B_{\beta(\sigma)}\right)\left(\partial_{\rho} B_{\gamma}^{(\tau)}\right) \eta_{\delta(\tau)}+\left(\partial^{\tau} B_{\beta(\sigma)}\right) \partial^{\rho}\left(B_{\gamma(\rho)} \eta_{\delta(\tau)}\right)\right)\right)+ \\
& \left(c_{2}\right)^{2} \varepsilon^{\alpha \beta \gamma \delta}\left(B_{\alpha}^{(\sigma)}\left(\partial^{\rho} B_{\beta(\sigma)}\right)\left(2\left(\partial^{\tau} B_{\gamma(\rho)}\right) \eta_{\delta(\tau)}+B_{\gamma(\rho)} \partial^{\tau} \eta_{\delta(\tau)}\right)+\right. \\
& \left.\left(A_{\alpha \beta(\sigma)}^{*} \partial^{\rho} B_{\gamma}^{(\sigma)}-\left(\partial^{\rho} A_{\alpha \beta(\sigma)}^{*}\right) B_{\gamma}^{(\sigma)}\right)\left(2\left(\partial^{\tau} B_{\delta(\rho)}\right) C_{(\tau)}+B_{\delta(\rho)} \partial^{\tau} C_{(\tau)}\right)\right) .(75)
\end{aligned}
$$

By inspecting the right hand-side of (75), we observe that all the terms proportional with $\left(c_{1}\right)^{2}$ simply reduce to a four-dimensional divergence, while those with $\left(c_{2}\right)^{2}$ and $c_{1} c_{2}$ cannot be written as in (74). Then the consistency of $\stackrel{(1)}{S}$ requires that

$$
c_{2}=0
$$


Under these considerations, (29) is satisfied for $\stackrel{(2)}{S}=0$. The remaining equations involved with the higher-order deformations hold if we set $\stackrel{(k)}{S}=0, k>2$. In addition, the constant $c_{1}$ takes any non-zero real value, and, for definiteness, will be fixed to unity

$$
c_{1}=1
$$

\section{Identification of the interacting theory}

Now, we are in the position to identify the interacting theory. The complete deformed solution to the master equation for the model under study, which is consistent to all orders in the deformation parameter, reads

$$
\begin{aligned}
& \bar{S}=\int d^{4} x\left(\frac{1}{2}\left(-B_{\alpha}^{(\sigma)} B_{(\sigma)}^{\alpha}+A_{(\rho)}^{\alpha \beta} \bar{H}_{\alpha \beta}{ }^{(\rho)}\right)+\right. \\
& \varepsilon^{\alpha \beta \gamma \delta}\left(A_{\alpha \beta}^{*{ }^{(\sigma)}}\left(D_{\gamma}\right)_{\sigma}{ }_{\sigma}-g \partial_{\sigma} A_{\alpha \beta}^{*}{ }^{(\rho)} B_{\gamma}^{(\sigma)}\right) \eta_{\delta(\rho)}+ \\
& \left(\eta^{* \alpha(\sigma)}\left(D_{\alpha}\right)_{\sigma}^{\rho}-g\left(\partial_{\sigma} \eta^{* \alpha(\rho)}\right) B_{\alpha}^{(\sigma)}\right) C_{(\rho)}- \\
& \left.g \varepsilon^{\alpha \beta \gamma \delta} A_{\alpha \beta}^{*{ }^{(\sigma)}}\left(\partial_{\sigma} A_{\gamma \delta}^{*}{ }^{(\rho)}\right) C_{(\rho)}\right),
\end{aligned}
$$

where the field strength components of $B_{\alpha}^{(\rho)}$ are deformed like

$$
\bar{H}_{\alpha \beta}{ }^{(\rho)}=H_{\alpha \beta}{ }^{(\rho)}-g\left(\partial_{\sigma} B_{[\alpha}^{(\rho)}\right) B_{\beta]}^{(\sigma)},
$$

and the "covariant derivative" is defined via

$$
\left(D_{\gamma}\right)_{\sigma}^{\rho}=\delta_{\sigma}^{\rho} \partial_{\gamma}+g \partial_{\sigma} B_{\gamma}^{(\rho)}
$$

The deformed solution (78) contains all the information on the gauge structure of the resulting interacting theory. More precisely, the terms of antighost number zero induce the Lagrangian action of the coupled model,

$$
\bar{S}_{0}\left[A_{(\sigma)}^{\alpha \beta}, B_{\alpha}^{(\sigma)}\right]=\frac{1}{2} \int d^{4} x\left(-B_{\alpha}^{(\sigma)} B_{(\sigma)}^{\alpha}+A_{(\rho)}^{\alpha \beta} \bar{H}_{\alpha \beta}^{(\rho)}\right),
$$

while the pieces of antighost number one furnish its gauge transformations

$$
\begin{aligned}
& \bar{\delta}_{\epsilon} A_{(\sigma)}^{\alpha \beta}=\varepsilon^{\alpha \beta \gamma \delta}\left(\left(D_{\gamma}\right)_{\sigma}^{\rho}+g \delta_{\sigma}^{\rho}\left(\partial_{\tau} B_{\gamma}^{(\tau)}+B_{\gamma}^{(\tau)} \partial_{\tau}\right)\right) \epsilon_{\delta(\rho)} \equiv \\
& \bar{R}_{(\sigma)}^{\alpha \beta}{ }_{(\rho)} \epsilon_{\delta(\rho)}, \bar{\delta}_{\epsilon} B_{\alpha}^{(\sigma)}=0,
\end{aligned}
$$


where the new gauge generators of the tensor fields of degree three are expressed by

$$
\bar{R}_{(\sigma)}^{\alpha \beta \delta(\rho)}=\varepsilon^{\alpha \beta \gamma \delta}\left(\left(D_{\gamma}\right)_{\sigma}^{\rho}+g \delta_{\sigma}^{\rho}\left(\partial_{\tau} B_{\gamma}^{(\tau)}+B_{\gamma}^{(\tau)} \partial_{\tau}\right)\right) .
$$

The elements with antighost number two that are simultaneously linear in the ghosts for ghosts and in the antifields of the ghosts determine the first-order reducibility functions

$$
\bar{Z}_{\alpha(\sigma)}^{(\rho)}=\left(D_{\alpha}\right)_{\sigma}^{\rho}+g \delta_{\sigma}^{\rho}\left(\partial_{\tau} B_{\alpha}^{(\tau)}+B_{\alpha}^{(\tau)} \partial_{\tau}\right)
$$

The appearance of the terms quadratic in the antifields of the fields $A_{(\sigma)}^{\alpha \beta}$ and linear in the ghosts for ghosts signifies that the first-order reducibility relations

$$
\bar{R}_{(\sigma)}^{\alpha \beta \delta(\rho)} \bar{Z}_{\delta(\rho)}^{(\tau)} \approx 0
$$

hold only on-shell (i.e., on the stationary surface of the field equations deriving from the action (81)), in contrast to the free model, for which the reducibility takes place off-shell. The absence of the antifields $B_{(\sigma)}^{* \alpha}$ emphasizes that the deformation procedure does not endow the auxiliary fields with gauge invariances. Meanwhile, the absence of pieces with antighost number two that are both quadratic in the ghosts $\eta_{\delta(\rho)}$ and linear in their antifields enables us to state that the gauge algebra of the interacting model remains abelian. Of course, we can always add to action (81) any polynomial in the auxiliary fields, without further deforming the gauge symmetry.

The added interactions do not spoil either the locality, or the manifest Lorentz covariance, and, essentially, are non-trivial as the terms involving antifields cannot be removed from the deformed solution to the maser equation by adding to it trivial ( $s$-exact modulo $d$ ) terms.

\section{Conclusion}

To conclude with, in this paper we have investigated the consistent Lagrangian interactions for a special class of covariant reducible mixed-symmetry type tensor gauge fields of degree three. In spacetime dimensions strictly greater than four the couplings do not modify the gauge symmetry of the initial free model, and are merely given by strictly gauge-invariant quantities or generalized Chern-Simons terms. A privileged situation is encountered 
in four spacetime dimensions, where there appear non-trivial consistent interactions that truly deform the gauge symmetry and the behaviour of the reducibility relations, but not the gauge algebra. In this sense, both situations reveal the rigidity of the original abelian gauge algebra against the deformation procedure.

The analysis developed in this paper can be useful at the study of introducing general interactions among covariant mixed-symmetry type tensor gauge fields, such as those involved with integer higher spin gauge theories (in direct or dual formulations).

\section{Acknowledgments}

This work has been supported by MEC-CNCSIS-Romania (type-A grant 944/2002).

\section{References}

[1] A. Barkallil, G. Barnich and C. Schomblond, Results on the Wess-Zumino consistency condition for arbitrary Lie algebras math-ph/0205047

[2] G. Barnich, Refining the anomaly consistency condition, Phys. Rev. D62 (2000) 045007 hep-th/0003135

[3] G. Barnich, F. Brandt and M. Henneaux, Conserved currents and gauge invariance in Yang-Mills theory, Phys. Lett. B346 (1995) 81-86 hep-th/9411202

[4] G. Barnich, F. Brandt and M. Henneaux, General solution of the WessZumino consistency condition for Einstein gravity, Phys. Rev. D51 (1995) 1435-1439 hep-th/9409104

[5] M. Dubois-Violette, M. Henneaux, M. Talon and C. M. Viallet, General Solution of the Consistency Equation, Phys. Lett. B289 (1992) 361-367 hep-th/9206106

[6] G. Barnich, A note on the BRST cohomology of the extended antifield formalism, Proceedings of the "Spring School in QFT, Supersymmetries 
and Superstrings" (Calimanesti, Romania, 24-30 April 1998), Phys. Ann. Univ. of Craiova 9 (1999) 92-106 hep-th/9912247

[7] G. Barnich, F. Brandt and M. Henneaux, Local BRST cohomology in gauge theories, Phys. Rept. 338 (2000) 439-569 hep-th/0002245

[8] G. Barnich, Higher order cohomological restrictions on anomalies and counterterms, Phys. Lett. B419 (1998) 211-216 hep-th/9710162

[9] M. Henneaux, Anomalies and Renormalization of BFYM Theory, Phys. Lett. B406 (1997) 66-69 hep-th/9704023

[10] G. Barnich and M. Henneaux, Renormalization of gauge invariant operators and anomalies in Yang-Mills theory, Phys. Rev. Lett. 72 (1994) 1588-1591 hep-th/9312206

[11] G. Barnich, A general non renormalization theorem in the extended antifield formalism, JHEP 9903 (1999) 010 hep-th/9805030

[12] M. Henneaux, Remarks on the renormalization of gauge invariant operators in Yang-Mills theory, Phys. Lett. B313 (1993) 35-40 hep-th/9306101; Erratum-ibid. B316 (1993) 633

[13] G. Barnich and F. Brandt, Covariant theory of asymptotic symmetries, conservation laws and central charges, Nucl. Phys. B633 (2002) 3-82 hep-th/0111246

[14] G. Barnich, Classical and quantum aspects of the extended antifield formalism, These d'agregation, Universite Libre de Bruxelles (June 2000) hep-th/0011120

[15] F. Brandt, M. Henneaux and A. Wilch, Extended antifield-formalism, Nucl. Phys. B510 (1998) 640-656 hep-th/9705007

[16] F. Brandt, M. Henneaux and A. Wilch, Global Symmetries in the Antifield-Formalism, Phys. Lett. B387 (1996) 320-326 hep-th/9606172

[17] M. Henneaux, Consistent Interactions Between Gauge Fields: The Cohomological Approach, Contemp. Math. 219 (1998) 93 hep-th/9712226

[18] M. Henneaux and B. Knaepen, All consistent interactions for exterior form gauge fields, Phys. Rev. D56 (1997) 6076-6080 hep-th/9706119 
[19] M. Henneaux, B. Knaepen and C. Schomblond, Characteristic cohomology of p-form gauge theories, Commun. Math. Phys. 186 (1997) 137-165, hep-th/9606181

[20] M. Henneaux, Uniqueness of the Freedman-Townsend Interaction Vertex For Two-Form Gauge Fields, Phys. Lett. B368 (1996) 83-88 hep-th/9511145

[21] G. Barnich and M. Henneaux, Consistent couplings between fields with a gauge freedom and deformations of the master equation, Phys. Lett. B311 (1993) 123-129 hep-th/9304057

[22] N. Boulanger, Multi-graviton theories : yes-go and no-go results, Fortsch. Phys. 50 (2002) 858-863 hep-th/0111216

[23] N. Boulanger, T. Damour, L. Gualtieri and M. Henneaux, Inconsistency of interacting, multi-graviton theories, Nucl. Phys. B597 (2001) 127-171 hep-th/0007220

[24] N. Boulanger, T. Damour, L. Gualtieri and M. Henneaux, No consistent cross-interactions for a collection of massless spin-2 fields, Proceedings of the "Spring School in QFT and Hamiltonian Systems" (Calimanesti, Romania, 2-7 May 2000), Phys. Ann. Univ. of Craiova 10 (2000) 94-106 hep-th/0009109

[25] G. Barnich, F. Brandt and M. Grigoriev, Seiberg-Witten maps and noncommutative Yang-Mills theories for arbitrary gauge groups, JHEP 0208 (2002) 023 hep-th/0206003

[26] G. Barnich, F. Brandt and M. Grigoriev, Seiberg-Witten maps in the context of the antifield formalism, Fortsch. Phys. 50 (2002) 825-830 hep-th/0201139

[27] G. Barnich, M. Grigoriev and M. Henneaux, Seiberg-Witten maps from the point of view of consistent deformations of gauge theories, JHEP 0110 (2001) 004 hep-th/0106188

[28] M. Dubois-Violette and M. Heneaux, Tensor fields of mixed Young symmetry type and N-complexes, Commun. Math. Phys. 226 (2002) 393-418 math.QA/0110088 
[29] M. Dubois-Violette and M. Heneaux, Generalized cohomology for irreducible tensor fields of mixed Young symmetry type, Lett. Math. Phys. 49 (1999) 245-252 math.QA/9907135

[30] X. Bekaert and N. Boulanger, Tensor gauge fields in arbitrary representations of $G L(D, R)$ : duality \& Poincare lemma hep-th/0208058

[31] P. de Medeiros and C. Hull, Exotic tensor gauge theory and duality hep-th/0208155

[32] X. Bekaert N. Boulanger and M. Henneaux, Consistent deformations of dual formulations of linearized gravity : a no-go result hep-th/0210278

[33] E. Cremmer and J. Scherk, Spontaneous dynamical breaking of gauge symmetry in dual models, Nucl. Phys. B72 (1974) 117-124

[34] M. Kalb and P. Ramond, Classical direct interstring action, Phys. Rev. D9 (1974) 2273-2284

[35] C. Teitelboim, Monopoles of higher rank, Phys. Lett. B167 (1986) 69

[36] C. Teitelboim, Gauge invariance for extended objects, Phys. Lett. B167 (1986) 63

[37] A. Lahiri, Constrained dynamics of decoupled abelian two-form, Mod. Phys. Lett. A8 (1993) 2403-2412 hep-th/9302046

[38] M. B. Green, J. H. Schwarz and E. Witten, Superstring theory, Cambridge University Press, Cambridge (1987)

[39] E. Witten, Noncommutative geometry and string field theory, Nucl. Phys. B268 (1986) 253

[40] D. Z. Freedman and P. K. Townsend, Antisymmetric tensor gauge theories and non-linear $\sigma$-models, Nucl. Phys. B177 (1981) 282-296

[41] G. Barnich, F. Brandt and M. Henneaux, Local BRST cohomology in the antifield formalism: I. General theorems, Commun. Math. Phys. 174 (1995) 57-92 hep-th/9405109

[42] I. A. Batalin and G. A. Vilkovisky, Gauge algebra and quantization, Phys. Lett. B102 (1981) 27 
[43] I. A. Batalin and G. A. Vilkovisky, Feynman rules for reducible gauge theories, Phys. Lett. B120 (1983) 166

[44] I. A. Batalin and G. A. Vilkovisky, Quantization of gauge theories with linearly dependent generators, Phys. Rev. D28 (1983) 2567; Erratumibid. D30 (1984) 508

[45] I. A. Batalin and G. A. Vilkovisky, Closure of the gauge algebra, generalized Lie algebra equations and Feynman rules, Nucl. Phys. B134 (1984) 106

[46] I. A. Batalin and G. A. Vilkovisky, Existence theorem for gauge algebra, J. Math. Phys. 26 (1985) 172

[47] M. Henneaux, Lectures on the antifield-BRST formalism for gauge theories, Nucl. Phys. B (Proc. Suppl.) 18A (1990) 47-106

[48] M. Henneaux and C. Teitelboim, Quantization of Gauge Systems, Princeton University Press, Princeton, New Jersey (1992)

[49] J. Gomis, J. Paris and S. Samuel, Antibracket, Antifields and GaugeTheory Quantization, Phys.Rept. 259 (1995) 1-145 hep-th/9412228

[50] G. Barnich, F. Brandt and M. Henneaux, Local BRST cohomology in the antifield formalism: II. Application to Yang-Mills theory, Commun. Math. Phys. 174 (1995) 93-116 hep-th/9405194 